\title{
Silencing long non-coding RNA HOTAIR exerts anti-oncogenic effect on human acute myeloid leukemia via demethylation of HOXA5 by inhibiting Dnmt3b
}

\author{
Si-Li Wang ${ }^{1,2^{*}}$, Yun Huang ${ }^{1}$, Rui Su ${ }^{1}$ and Yong-Yang Yu ${ }^{3}$
}

\begin{abstract}
Background: As an aggressive hematological malignancy, acute myeloid leukemia (AML) remains a dismal disease with poor prognosis. Long non-coding RNAs (IncRNAs) have been widely reported to be involved in tumorigenesis of AML. Here, we define an important role of IncRNA HOTAIR in AML in relation to HOXA5 methylation.

Methods: Firstly, the expression of HOTAIR was examined in AML samples and cells collected. Next, gain- or loss-of function experiments were conducted in AML cells to explore the effect of HOTAIR on AML. Then, relationship among HOXA5 promoter methylation, HOTAIR and Dnmt3b was measured. Expression of HOXA5 and cell proliferation/apoptosis-related genes was also detected. A last, in vivo assay was performed to assess the tumor formation in nude mice in order to explore the roles of HOTAIR and HOXA5 in cell apoptosis and proliferation.
\end{abstract}

Results: LncRNA HOTAIR was found to be upregulated in AML cells and tissues. With silencing of HOTAIR and overexpression of HOXA5, AML cell proliferation was decreased while the apoptosis was induced. Furthermore, HOTAIR was observed to recruit Dnmt3b and to increase HOXA5 promoter methylation. Moreover, silencing HOTAIR and upregulating HOXA5 were found to induce apoptosis and reduce proliferation of AML cells in vivo.

Conclusion: Our findings highlight the anti-tumor ability of HOTAIR silencing in AML, suggesting that silencing HOTAIR was able to inhibit AML progression through HOXA5 promoter demethylation by decreasing Dnmt3b.

Keywords: Acute myeloid leukemia, Long noncoding RNA HOTAIR, HOXA5, Dnmt3b, Promoter methylation, Proliferation, Apoptosis

\section{Background}

As the most common type of blood disease, acute myelogenous leukemia (AML) is highly prevalent in adults with a high relapse rate [1]. The incidence of AML is approximate 1.3 per 100,000 for people under 60 years old and 12.2 per 100,000 for people over 65 years old [2]. AML is characterized by a decrease in the proliferation of functional blood cells and excessive proliferation and accumulation of immature leukemic blasts [3]. AML

\footnotetext{
*Correspondence: Drwangsili_WSL@163.com

1 Department of Hematology, The First Affiliated Hospital of Xiamen University, No. 55, Zhenhai Road, Xiamen 361003, Fujian, People's Republic of China

Full list of author information is available at the end of the article
}

has been reported to be caused by diverse factors, such as cytogenetic risk, age, white blood cell count, and fetal liver tyrosine kinase 3 internal tandem duplication (FLT3 ITD) $[4,5]$. In spite of the improvement achieved in controlling AML, AML still leads to low overall survival rates with poor prognosis [6]. Therefore, it is in urgent need to find out more effective biomarkers for AML to strengthen the diagnosis and prognosis of AML.

It has been widely revealed that long non-coding RNAs (lncRNAs), a family of non-protein-coding transcripts with the length of over $200 \mathrm{nt}$ act pivotal functions in blood diseases [7]. Several lncRNAs have been demonstrated to function in AML, including CCDC26, KCNQ1 overlapping transcript 1 (KCNQ1OT1), and homeobox 
antisense intergenic RNA myeloid 1 (HOTAIRM1) [810]. More specifically, homeobox antisense intergenic RNA (HOTAIR) serves as an underlying predictor for the relapse diagnosis and poor prognosis in patients with AML [11]. Furthermore, the highly expressed HOTAIR was suggested to be associated with the progression of AML via the regulation of cell proliferation and apoptosis [12]. Furthermore, Up-regulation of HOTAIR and methylation of HOXA5 were found during the development of breast cancer [13] and a previous study indicated that HOTAIR and homeobox A5 (HOXA5) worked together and were closely correlated to growth and metastasis of non-small cell lung cancer [14]. HOXA5, a member belonging to the HOX gene family, could modulate the differentiation of epithelial and hematopoietic cells, and HOXA5 methylation was linked to disease aggressiveness [15]. Silencing HOXA5 has been reported to reduce cell proliferation in AML patients [16]. Dnmt3b was reported to encode a DNA methyltransferase, which is involved in aberrant epigenetic changes that lead to leukemia [17]. Elevated transcript levels of DNA methyltransferase $3 \mathrm{~b}$ (Dnmt3b) and upregulated promoter methylation of HOXA5 were found to be associated with the developmental processes of estrogen receptor negative breast tumors [18]. The present study aims to dig out the specific mechanism by which lncRNA HOTAIR and HOXA5 methylation influences the proliferation and apoptosis of AML cells.

\section{Materials and methods \\ Ethics statement}

The present study was conducted in strict accordance to the protocols approved by the Institutional Review Board of The First Affiliated Hospital of Xiamen University. Written informed consent documentations were signed by all participating patients. All animal experiments were performed in full compliance with the national principles for animal usage in research with the approval of the Animal Care and Use Committee. All animal experiments in this study were in conformity to the guidelines of management and use of local laboratory animals and approved by the Guide for the Care and Use of Laboratory Animal issued by the National Institutes of Health.

\section{Study subjects}

A total of 90 patients diagnosed with AML in The First Affiliated Hospital of Xiamen University from May 2016 to September 2017 were enrolled in this study, including 47 males and 43 females aged from 22 to 65 years old with the median age of 45 years old. All patients met the World Health Organization (WHO) diagnostic criteria and were diagnosed by bone marrow cell morphology test including Wright-Giemsa staining, cytochemical staining [peroxidase (POX), alpha-naphthyl-acetate-esterase (ANAE), specific esterase (CE), and periodic acid-Schiff (PAS)] and immunophenotype test (flow cytometry). Patients with combined severe infection, other solid tumors or immune system diseases were excluded. Meanwhile, 30 normal human bone marrow samples were selected as experimental controls. The clinical characteristics of enrolled patients are shown in Additional file 1: Table S1.

\section{Cell treatment}

Human leukemia cell lines (HL-60, K562, THP-1 and U937) from American Type Culture Collection (Manassas, VA, USA) were cultured in RPMI-1640 medium (Gibco, Carlsbad, California, USA) conjugated with $10 \%$ fetal bovine serum (FBS), $10 \mathrm{ug} / \mathrm{mL}$ streptomycin and $100 \mathrm{U} / \mathrm{mL}$ penicillin in an incubator (Thermo Fisher Scientific Inc., Waltham, Massachusetts, USA) at $37^{\circ} \mathrm{C}$ with $5 \% \mathrm{CO}_{2}$. Hematopoietic stem cells were isolated from normal bone marrow samples, and reverse transcription quantitative polymerase chain reaction (RT-qPCR) was conducted to measure the level of HOTAIR in HL-60, K562, THP-1, U937 cells and normal bone marrow samples [19]. Cells in the logarithmic growth stage were detached by trypsin and inoculated into a 6-well plate with a density rate of $1 \times 10^{5}$ cells/well. When the cell confluence reached about $75 \%$, cells were cultured with the following plasmids according to the instructions of Lipofectamine 2000 (Invitrogen Inc., Car, Cal, USA): shnegative control (NC) (5'-CUACAACAGCCACAACGU CdTdT-3'), sh-HOTAIR-1 (sense: 5'-GAACGGGAG UACAGAGAGAUU- $3^{\prime}$; antisense: $3^{\prime}$-UUCUUGCCC UCAUGUCUCUCU-5'), sh-HOTAIR-2 (sense: $5^{\prime}$-CCA CAUGAACGCCCAGAGAUU-3'; antisense: $3^{\prime}$-UUG GUGUACUUGCGGGUCUCU-5'), and sh-HOTAIR-3 (sense: 5'-UAACAAGACCAGAGAGCUGUU-3'; antisense: $3^{\prime}$-UUAUUGUUCUGGUCUCUCGAC-5'). After culturing for $48 \mathrm{~h}$, the silencing efficiency of shRNA was detected by RT-qPCR.

\section{RT-qPCR}

Total RNA was extracted using the Trizol kit (15596026, Invitrogen Inc., Car, Cal, USA). Based on the instructions of Primescript RT reagent kit (RR047A, TaKaRa, Tokyo, Japan), RNA was reversely transcribed into complementary DNA (cDNA). RT-qPCR was then carried out using the Fast SYBR Green PCR Kit (Applied Biosystems, Carlsbad, CA, USA) on the ABI PRISM 7300 RT-PCR System (Applied Biosystems, Carlsbad, CA, USA). The reaction conditions were as follows: pre-denaturation at $95{ }^{\circ} \mathrm{C}$ for $5 \mathrm{~min}, 40$ cycles of denaturation at $95{ }^{\circ} \mathrm{C}$ for $30 \mathrm{~s}$, annealing, and extension at $60{ }^{\circ} \mathrm{C}$ for $1 \mathrm{~min}$. Each sample was set with 3 duplicated 
wells. Glyceraldehyde-3-phosphate dehydrogenase (GAPDH) gene served as an internal reference. The relative expression of HOTAIR and HOXA5 between the experiment group and the control group was calculated based on the $2^{-\Delta \Delta \mathrm{Ct}}$ method. The primers sequences are shown in Table 1.

\section{Western blot analysis}

After cell collection, the cells were lysed with an enhanced radioimmunoprecipitation assay (RIPA) lysis buffer (Boster Biological Technology, Co., Ltd., Wuhan, China) containing protease inhibitor. Protein concentration was measured using a bicinchoninic acid (BCA) protein assay kit (Boster Biological Technology, Co., Ltd., Wuhan, China). The protein was separated by $10 \%$ sodium dodecyl sulfate-polyacrylamide gel electrophoresis (SDS-PAGE) and transferred onto the polyvinylidene fluoride (PVDF) membranes. After being blocked with 5\% bovine serum albumin (BSA) for $2 \mathrm{~h}$ at room temperature, the membranes were incubated with the addition of diluted primary rabbit antibodies (Abcam Inc., Cambridge, MA, USA) against HOXA5 (ab82645, 1:500), Bcl-2 Associated X (Bax, ab32503, 1:1000), Bcl-2 (ab32124, 1:500), MCP-1 (ab9669, 1:500), cleaved-caspase3 (ab49822, 1:500), p27 (ab32034, 1:5000), and cyclin G (ab170389, 1:100) at $4{ }^{\circ} \mathrm{C}$ overnight. After being washed three times with phosphate buffered saline-Tween 20 (PBST), the membranes were incubated after the addition of horseradish peroxidase (HRP)-labeled goat anti-rabbit secondary antibody (ab205719; 1:2000, Abcam Inc., Cambridge, MA, USA) at room temperature for $1 \mathrm{~h}$. Then the membranes were washed three times with PBST and detected using the enhanced chemiluminescence (ECL, EMD Millipore Corporation, Billerica, MA, USA). Gray-value quantification of bands in western blot images was performed using Image J analysis software, and GAPDH was taken as an internal reference. The experiment was repeated three times.

\section{Table 1 Primer sequence for RT-qPCR}

\begin{tabular}{ll}
\hline Genes & Primer sequence \\
\hline HOTAIR & F: 5'-CAGTGGGGAACTCTGACTCG-3' \\
& R: 5'-GTGCCTGGTGCTCTCTTACC-3' \\
HOXA5 & F: 5'-CGCCCAACCCCAGATCTA-3' \\
& R: 5'-GGCCGCCTATGTTGTCATG-3' \\
GAPDH & F: 5'-GCCAAGGTCATCCATGACAACT-3' \\
& R: 5'-GAGGGGCCATCCACAGTCTT-3' \\
\hline
\end{tabular}

$R T-q P C R$ reverse transcription quantitative polymerase chain reaction, HOTAIR Hox transcript antisense intergenic RNA, HOXA5 homeobox A5, GAPDH glyceraldehyde-3-phosphate dehydrogenase, $F$ forward, $R$ reverse

\section{Fluorescence in situ hybridization (FISH)}

The location of HOTAIR in AML cells was detected by FISH according to the instructions of RiboTM lncRNA FISH Probe Mix (Red) (Guangzhou RiboBio Co., Ltd., Guangzhou, China). AML cells were cultured in 6-well plates, which were coated with coverslips for $1 \mathrm{~d}$ until the cell confluence reached about $80 \%$. After that, cells were washed with phosphate-buffered saline (PBS), fixed with $1 \mathrm{~mL}$ of $4 \%$ paraformaldehyde at room temperature. After being treated with $2 \mu \mathrm{g} / \mathrm{mL}$ protease $\mathrm{K}$, glycine and acetylation reagents, cells were incubated with $250 \mu \mathrm{L}$ of pre-hybridization solution at $42{ }^{\circ} \mathrm{C}$ for $1 \mathrm{~h}$. After the pre-hybridization solution was aspirated, cells were added with $250 \mu \mathrm{L}$ of hybridization solution containing $300 \mathrm{ng} / \mathrm{mL}$ HOTAIR probe and then hybridized overnight at $42{ }^{\circ} \mathrm{C}$. After washing with PBST three times, the cells were stained with 4',6-diamidino2-phenylindole (DAPI) (1:800) diluted with PBST for 5 min, rinsed with PBST three times (3 min each time) and sealed with anti-fluorescent quencher. Five different fields were selected and photographed under a fluorescence microscope (Olympus Co., Ltd., Tokyo, Japan). Each experiment was repeated three times.

\section{Methylation specific PCR (MS-PCR)}

Based on the DNA Methylation-Gold ${ }^{\mathrm{TM}}$ Kit (D5005, Zymo Research, Irvine, CA, USA), the methylation level of the HOXA5 promoter region was measured. The primer sequences for methylation reaction were HOXA5-MD (5'-TTTAGCGGTGGCGTTCG-3 ${ }^{\prime}$ ) and HOXA5-MR (5'-ATACGACTTCGAATCACGTA-3'), and the primer sequences for the un-methylation reaction were HOXA5-UD (5'-TTGGTGAAGTTGGGTG$\left.3^{\prime}\right)$ and HOXA5-UR (5'-AATACAACTTCAAATCAC ATAC- $\left.3^{\prime}\right)$. The purified DNA was added into cytosine to thymine $(\mathrm{CT})$ conversion reagent for denaturation and bisulfite conversion. Then the desulfurization and purification were conducted using a reaction column, and the purified DNA could be used for subsequent PCR reaction. The PCR reaction conditions were as follows: pre-denaturation at $95{ }^{\circ} \mathrm{C}$ for $10 \mathrm{~min}$, and 35 cycles of denaturation at $95{ }^{\circ} \mathrm{C}$ for $45 \mathrm{~s}$, methylation at $56{ }^{\circ} \mathrm{C}$ for $45 \mathrm{~s}$, non-methylation at $45^{\circ} \mathrm{C}$ for $45 \mathrm{~s}$, extension at $72{ }^{\circ} \mathrm{C}$ for $45 \mathrm{~s}$, and a final elongation at $72{ }^{\circ} \mathrm{C}$ for $10 \mathrm{~min}$. The reaction products subsequently underwent agarose gel electrophoresis, which were then analyzed by imaging analysis. Each experiment was repeated three times.

\section{Dual luciferase reporter assay}

HOXA 5 promoter region was detected by dual luciferase reporter assay. Cells were inoculated into the 24-well 
plates and cultured with plasmids using Lipofectamine 2000 when cells confluence reached $60-80 \%$. The cells were collected after 24-48 h, rinsed with PBS three times and lysed with $75 \mu \mathrm{L}$ lysate at room temperature for 15-20 min, shaken every several min so that the cells could be completely covered with lysate. After collection of the cell lysate, luciferase activities were immediately detected based on the instructions of Dual luciferase assay kit with a luminometer (Monolight 2010; Analytical Luminescence Laboratory, San Diego, CA, USA). During the experiment, the thymidine kinase promoter-renilla luciferase reporter plasmid (pRL-TK) was used as the internal reference, the reaction system of firefly luciferase reaction system was LAR II, and the renilla luciferase reaction system was Stop\&Glo Reagent. The fluorometer was preheated, and the parameters were set. Then the determination started after each delay of $2 \mathrm{~s}$, with the determination time set as $10 \mathrm{~s}$. After adding with $100 \mu \mathrm{L}$ LAR II, the fluorescent tube was added with $20 \mu \mathrm{L}$ of cell lysate. After mixing for 2-3 times with a pipette tip, the fluorometer was placed into the fluorescent tube with reading started. Firefly luciferase reading was recorded and repeated once. A total of $100 \mu \mathrm{L}$ of Stop\&Glo Reagent was added into the same tube, and mixed. Then the fluorometer was placed into the fluorescent tube to record, and this step was repeated once. Each experiment was repeated three times.

\section{RNA immunoprecipitation (RIP)}

The binding of HOTAIR to Dnmt3b protein was detected using a RIP kit (Merck Millipore, Billerica, MA, USA). After being washed with pre-cooled and lysed with an equal volume of RIPA lysate (P0013B, Beyotime Institute of Biotechnology, Shanghai, China) in an ice bath for $5 \mathrm{~min}$, the cells were centrifuged at 14,000 rpm for 10 min at $4{ }^{\circ} \mathrm{C}$. Part of the cell extract was taken as input, and remaining cell extract was incubated with antibody for co-precipitation. A total of $50 \mu \mathrm{L}$ magnetic beads for each co-precipitation reaction system were washed and re-suspended with $100 \mu \mathrm{L}$ RIP Wash Buffer, and then $5 \mu \mathrm{g}$ of following antibody was added: rabbit anti Dmnt $3 \mathrm{~b}$ (1:100, ab2851, Abcam Inc., Cambridge, MA, UK) or rabbit anti-human immunoglobulin G (IgG) (1:100, ab109489, Abcam Inc., Cambridge, MA, UK) as an NC. The magnetic bead-antibody complex was washed, resuspended with $900 \mu \mathrm{L}$ RIP Wash Buffer, and incubated with $100 \mu \mathrm{L}$ cell extract overnight at $4{ }^{\circ} \mathrm{C}$. The samples were washed 3 times and placed on magnetic pedestal to collect the magnetic bead-protein complexes. The collected samples and input were separately digested by proteinase $\mathrm{K}$ to extract RNA for detecting HOTAIR in subsequent RT-qPCR.

\section{RNA pull-down assay}

AML cells were respectively transduced with wild-type (WT) biotinylated HOTAIR and mutant (MUT) biotinylated HOTAIR (50 nM each). After $48 \mathrm{~h}$ of treatment, the cells were washed with PBS and lysed with specific cell lysis (Ambion, Austin, Texas, USA) for $10 \mathrm{~min}$. After that, the lysate was cultured with M-280 streptavidin magnetic beads (Sigma, St. Louis, MO, USA) precoated with RNase-free bovine serum albumin (BSA) and yeast tRNA (Sigma, St. Louis, MO, USA) at $4{ }^{\circ} \mathrm{C}$ for $3 \mathrm{~h}$, washed twice with cold lysis buffer, and then washed with the low-salt buffer three times and with high-salt buffer once. Total protein was extracted with high-efficiency RIPA lysate, followed by determination of the level of Dnmt3b using western blot analysis. The experiment was repeated three times independently.

\section{Chromatin immunoprecipitation (CHIP)}

After fixed with $1 \%$ formaldehyde, AML cells were treated with ultrasonication. Cells were added with rabbit anti Dnmt3b (ab2851, 1:50, Abcam Inc., Cambridge, MA, UK) to bind to the Dnmt3b-HOXA5 promoter, and then added with Protein A Agarose/Salmon Sperm DNA. The precipitated complex of Dnmt3b antibody-Dnmt3bHOXA5 promoter was washed and eluted to obtain enriched Dnmt3b-HOXA5 promoter complex. After decrosslinked, the enriched HOXA5 promoter fragment was purified and subjected to PCR. The experiment was repeated three times independently.

\section{Flow cytometry}

AML Cells were collected, rinsed twice with cold PBS, and suspended with $400 \mu \mathrm{L} 1 \times$ Binding Buffer. Afterwards, the cell suspension was incubated with the addition of $5 \mu \mathrm{L}$ Annexin V-fluorescein isothiocyanate (FITC) at $4{ }^{\circ} \mathrm{C}$ for $15 \mathrm{~min}$ avoiding exposure to light. Added with $10 \mu \mathrm{L}$ propidium iodide (PI), the cells were then incubated at $4{ }^{\circ} \mathrm{C}$ avoiding exposure to light for $5 \mathrm{~min}$. Finally, the cells were analyzed by flow cytometry (BD FACS Calibur, Becton-Dickinson, San Jose, CA, USA) within $1 \mathrm{~h}$. The experiment was conducted three times independently.

\section{5-ethynyl-2'-deoxyuridine (EdU) assay}

The AML cells in the logarithmic growth stage were inoculated into the 24-well plates, with three duplicated wells set in each group. After that, EDU (C103411, Guangzhou RiboBio Co., Ltd., Guangzhou, China) was incubated with the cells for $2 \mathrm{~h}$ at a final concentration of $10 \mu \mathrm{mol} / \mathrm{L}$. After removal of the medium, cells were fixed in PBS implemented with $4 \%$ paraformaldehyde for $15 \mathrm{~min}$ at room temperature, washed twice 
with PBS conjugated with 3\% BSA, incubated with PBS containing $0.5 \%$ Triton- 100 for $20 \mathrm{~min}$ at room temperature, and then rinsed twice with PBS containing $3 \%$ BSA. And then, cells in each well were incubated with $100 \mu \mathrm{L}$ Apollo $^{\circledR} 567$ (Guangzhou RiboBio Co., Ltd., Guangzhou, China) at room temperature avoiding exposure to light for $30 \mathrm{~min}$, washed twice with PBS containing $3 \%$ BSA, and stained with $1 \times$ Hoechst 33,342 for $30 \mathrm{~min}$, followed by PBS wash 3 times. After cells were mounted, the number of EDU positive cells as well as the total cells in each field was observed and recorded under a fluorescence microscope (FM-600, Puda Optical Instrument Co., Ltd., Shanghai, China). The positive cells presented red. The experiment was performed three times independently.

\section{Tumor formation in nude mice}

A total of 64 BALB/c nude mice (5-7 weeks old, 18-22 g in weight, purchased from Lingchang Biotech Co., Ltd., Shanghai, China) were selected. The nude mice were housed under the specific pathogen-free (SPF) environment in Animal Experimental Center (Experimental Animal Qualification Certificate No. 257), with comfortable temperature and environment, aseptic feed and water and alternating $12 \mathrm{~h}$ light/dark cycles. The AML cell line was infected with lentivirus with a titer of $2 \times 10^{8} \mathrm{PFU} /$ $\mathrm{mL}$. After stably infected cell lines were obtained, $0.2 \mathrm{~mL}$ cell suspension with a density of $5 \times 10^{7}$ cells/ $\mathrm{mL}$ was inoculated into the subcutaneous space on the left side of the BALB/c nude mice with $1 \mathrm{~mL}$ syringe. A total of 55 mice were implanted with the following different cells for tumor formation with 11 mice in each group: the M-overexpression (oe)-NC (pLV-EGFP-NNC lentivirus-infected cells), M-oe-HOTAIR (pLVEGFP-N-HOTAIR lentivirus-infected cells), M-sh-NC (pLKO.1-shRN-NC lentivirus-infected cells), M-shHOTAIR (pLKO.1-HOTAIR-shRNA lentivirus-infected cells), and M-oe-HOTAIR + HOXA5 groups (pLV-EGFP$\mathrm{N}$-HOTAIR + pLV-EGFP-N-HOXA5 lentivirus-infected cells). After inoculation, all nude mice were housed in laminar-flow hoods in the SPF level animal room. After 4 weeks, the mice were euthanized by inhalation of carbon dioxide, and the xenografts were collected and weighed. Tumor measurements of each group were repeated three times. Then tumor tissue was fixed with $10 \%$ formalin and embedded in paraffin. Expression of Bax, cleaved-caspase3, p27, and cyclin G were detected by western blot analysis, and EdU assay was conducted to detect cell proliferation. Each experiment was repeated three times.

\section{Statistical analysis}

All data were processed by SPSS 21.0 statistical software (IBM Corp. Armonk, NY, USA). Measurement data were expressed as mean \pm standard deviation. Comparisons between two groups were conducted by means of $t$-test, and comparisons among multiple groups were assessed by one-way analysis of variance. A $p<0.05$ value indicated that the data were statistically significant.

\section{Results}

AML tissues and cells exhibit upregulated HOTAIR

Initially, the levels of HOTAIR in 90 patients with AML and 30 normal human bone marrow samples were measured. As shown in Fig. 1a, compared with the normal samples, HOTAIR was highly expressed in patients with AML $(p<0.001)$. Then, the HOTAIR level in different leukemia-associated cell lines was determined. The results showed that compared with the hematopoietic stem cells extracted from bone marrow, the expression of HOTAIR was significantly increased in U937, HL-60, THP-1 and K562 cell lines $(p<0.05)$, with HL-60 cell line exhibiting the highest level of HOTAIR (Fig. 1b). Therefore, the HL-60 cell line was selected for subsequent experiments. In conclusion, HOTAIR was upregulated in AML tissues and cells.

\section{Silencing HOTAIR leads to enhanced apoptosis and repressed proliferation of $\mathrm{HL}-60$ cells}

In order to find out the regulation of HOTAIR on apoptosis and proliferation of HL-60 cells, western blot analysis was conducted for the determination of the protein levels of apoptosis-related factors, including Bax, Bcl-2, MCP-1, cleaved-caspase3, p27, and cyclin G, and flow cytometry and EdU assay were performed to measure cell apoptosis and proliferation, respectively. As shown in Fig. 2a, compared with the sh-NC group, the expression of Bax, cleaved-caspase3, p27 and cyclin G in the sh-HOTAIR group was significantly increased while of MCP-1 and Bcl-2 was decreased $(p<0.05)$. In addition, compared with the sh-NC group, the apoptosis of HL-60 cells was notably increased in the sh-HOTAIR group $(p<0.05$; Fig. $2 \mathrm{~b})$. In comparison with the sh-NC group, the proportion of cells in the G0/G1 phases was elevated while proportion of cells in the $S$ phases was reduced in the sh-HOTAIR group (Fig. 2c). Then, EdU assay revealed that the proliferation of HL-60 cells in the sh-HOTAIR group was notably lower than that in the sh-NC group $(p<0.05$; Fig. $2 \mathrm{~d})$. These results suggested that silencing 

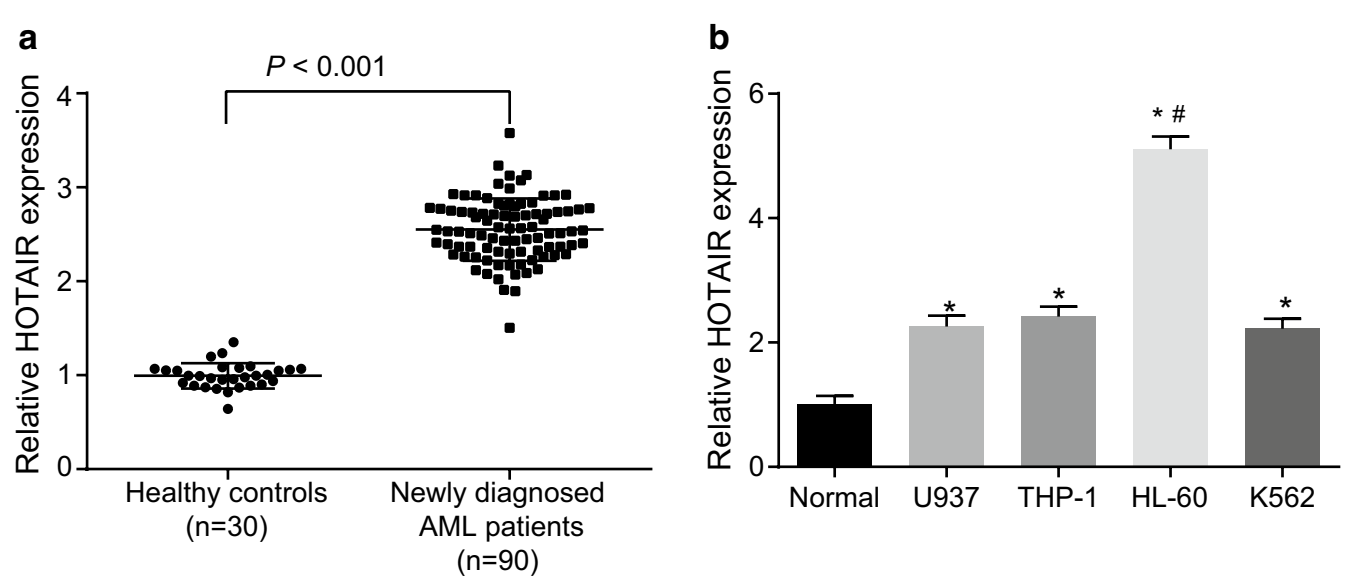

Fig. 1 HOTAIR is overexpressed in AML tissues and cells. a HOTAIR expression in AML samples examined by RT-qPCR. b HOTAIR expression in U937, $\mathrm{HL}-60, \mathrm{THP}-1$ and $\mathrm{K} 562$ cell lines and normal bone marrow cell line examined by RT-qPCR. ${ }^{*} p<0.05$ vs. the normal group; ${ }^{\#} p<0.05$ vs. the U937, THP-1 and K562 cell lines. RT-qPCR reverse transcription quantitative polymerase chain reaction, HOTAIR Hox transcript antisense intergenic RNA, NC negative control, AML acute myeloid leukemia. The results were measurement data. Comparisons between two groups were conducted by means of independent sample $t$-test, and comparisons among multiple groups were assessed by one-way analysis of variance. The experiment was independently repeated three times

of HOTAIR promoted apoptosis and inhibited proliferation of AML cells.

\section{Silencing HOTAIR promotes apoptosis and inhibits proliferation of $\mathrm{HL}-60$ cells by upregulating HOXA5} In order to investigate the effect of HOTAIR on HOXA5 expression in HL-60 cells, RT-qPCR was used to detect the expression of HOTAIR after silencing HOTAIR, which showed that the expression of HOTAIR in the sh-HOTAIR + sh-NC group was significantly lower than that in the sh-NC group $(p<0.05)$. In contrast to the sh-HOTAIR + sh-NC group, no obvious difference concerning the expression of HOTAIR was detected in the sh-HOTAIR+sh-HOXA5 group (Fig. 3a). Next, RT-qPCR and western blot analysis were performed to measure the level of HOXA5. As shown in Fig. 3b, c, compared with the sh-NC group, the HOXA5 expression was significantly increased in the sh-HOTAIR + sh-NC group, while decreased in the sh-HOTAIR + sh-HOXA group $(p<0.05)$, suggesting that silencing HOTAIR could upregulate HOXA5. In order to explore the role of HOXA5 in the apoptosis of HL-60 cells, western blot analysis was used to detect the expression of apoptosis-related factors. The results showed that compared with the sh-NC group, the sh-HOTAIR + sh-NC group revealed notably increased expression of Bax, cleavedcaspase3, p27, and cyclin $\mathrm{G}$ but decreased expression of MCP-1 and BCcl-2 $(p<0.05)$, while the opposite trend was observed in the sh-HOTAIR + sh-HOXA5 group $(p<0.05$; Fig. $3 \mathrm{~d})$. Then flow cytometry was adopted to assess the effects of HOXA5 on cell cycle distribution and apoptosis of HL-60 cells, which revealed that compared with the sh-NC group, the apoptosis and the proportion of HL-60 cells in the G0/G1 phase were significantly increased, and the proportion of cells in $S$ phase decreased in the sh-HOTAIR + sh-NC group $(p<0.05)$. Compared with the sh-HOTAIR + sh-NC group, the shHOTAIR + sh-HOXA5 group showed decreased apoptosis and proportion of cells in G0/G1 phase, but elevated proportion of cells in $\mathrm{S}$ phase $(p<0.05$; Fig. 3e, $\mathrm{f})$. Moreover, EDU was carried out for the detection of the effect of HOXA5 on the proliferation of HL-60 cells. As shown

\footnotetext{
(See figure on next page.)

Fig. 2 Silencing HOTAIR inhibits proliferation and promotes apoptosis of AML cells. a Protein expression of Bax, Bcl-2, MCP-1, cleaved-caspase3, p27 and cyclin G in HL-60 cells after interference with HOTAIR examined by western blot analysis. b Cell apoptosis of HL-60 cells after interference with HOTAIR examined by flow cytometry. c Cell cycle distribution of HL-60 cells after interference with HOTAIR detected by flow cytometry. d The proliferation of HL-60 cells after interference with HOTAIR examined by EdU assay ( $\times 200) .{ }^{*} p<0.05$ vs. the sh-NC group. RT-qPCR reverse transcription quantitative polymerase chain reaction, HOTAIR Hox transcript antisense intergenic RNA, HOXA5 homeobox A5, EDU 5-ethynyl-2'-deoxyuridine, NC negative control, AML acute myeloid leukemia, BCl-2 B-cell lymphoma 2, Bax BCl-2 associated X, MCP-1 monocyte chemoattractant protein 1. The results were measurement data. Comparisons between two groups were conducted by means of independent sample $t$-test. The experiment was independently repeated three times
} 


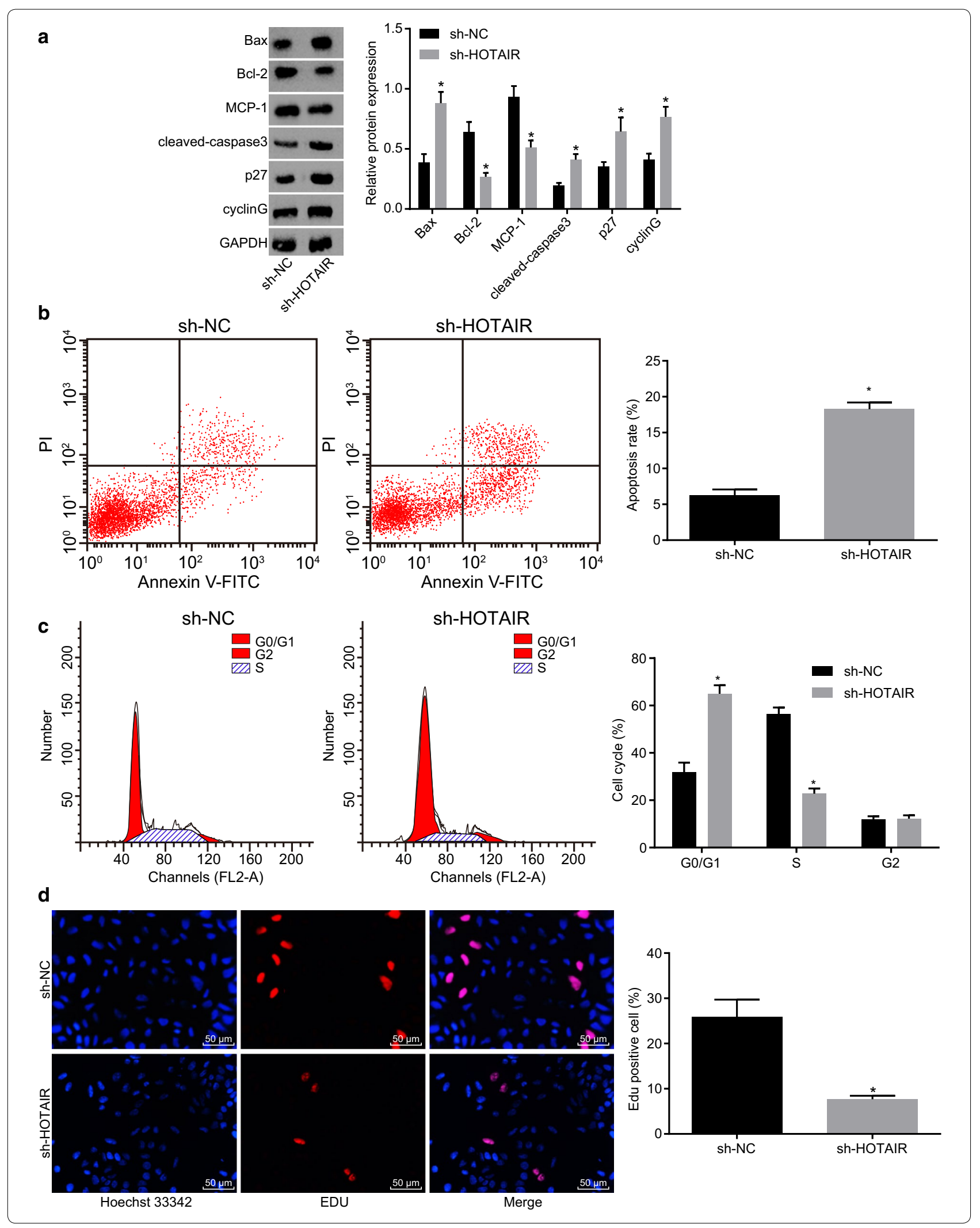




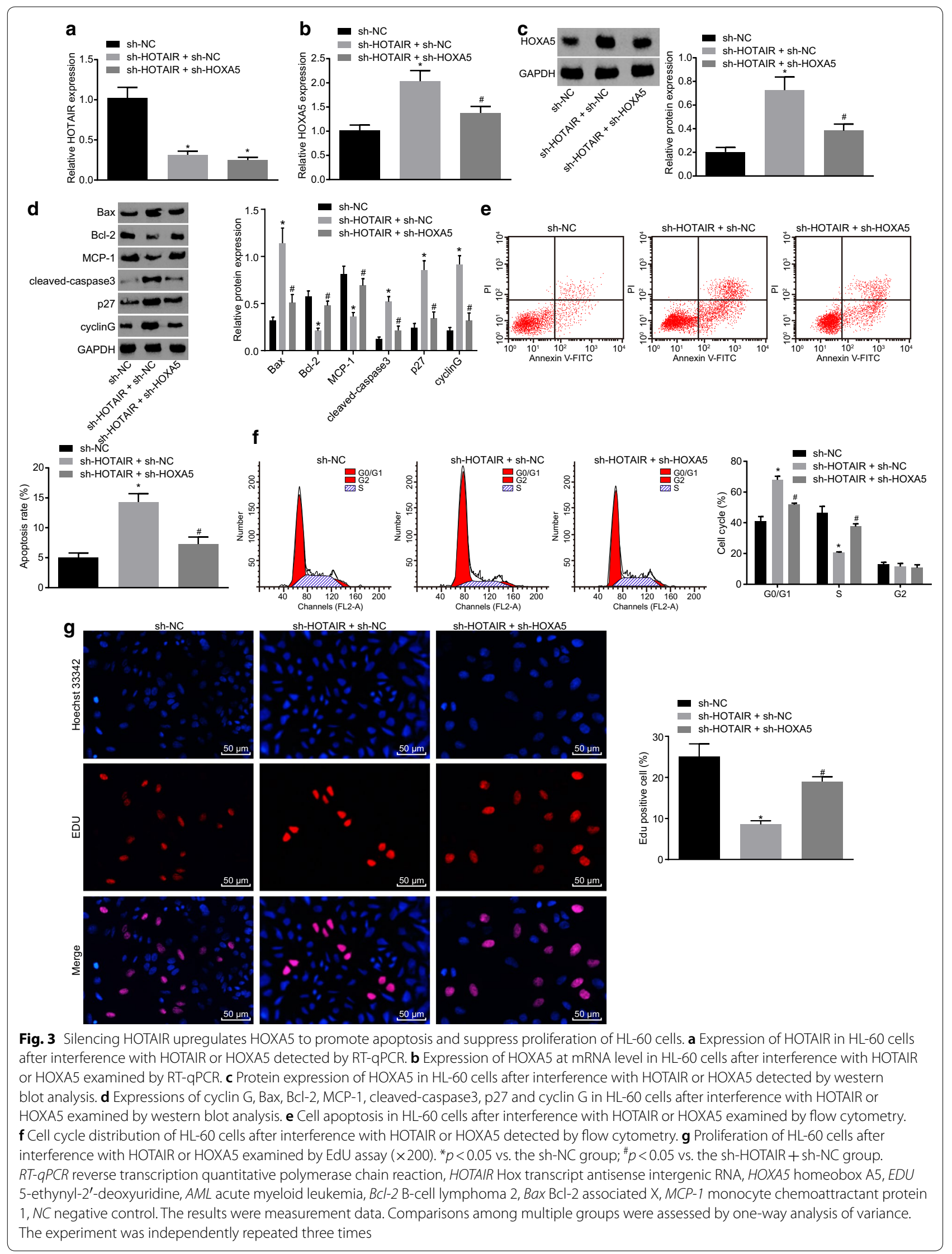


in Fig. 3g, compared with the sh-NC group, the proliferation of HL-60 cells was significantly decreased in the shHOTAIR + sh-NC group $(p<0.05)$. When compared with the sh-HOTAIR + sh-NC group, the sh-HOTAIR + shHOXA5 group showed notably elevated proliferation of HL-60 cells $(p<0.05)$. The above results demonstrated that silencing HOTAIR promoted the expression of HOXA5, thus promoting apoptosis and inhibiting proliferation of HL-60 cells.

\section{HOTAIR induces HOXA5 promoter methylation by binding Dnmt3b}

Subcellular localization of HOTAIR was predicated with the LncATLAS website (http://lncatlas.crg.eu/), which uncovered that HOTAIR existed primarily in the nucleus of multiple cell lines. Then FISH was employed to determine the subcellular localization of HOTAIR, finding that
HOTAIR was mainly distributed in the nucleus (Fig. 4a). Next, dual luciferase reporter assay was conducted to detect the binding of HOTAIR to HOXA5 promoter. As shown in Fig. 4b, compared with the oe-NC group, the luciferase activity of the oe-HOTAIR group was significantly decreased $(p<0.05$; Fig. 4b). High methylation level of HOXA5 was once found in AML samples, and HOXA5 methylation has been suggested to play an important role in the occurrence and development of AML [20]. A recent study found that Dnmt3b-mediated DNA methylation acted an essential role in the development of leukemia [21]. So we would like to explore whether HOTAIR and Dnmt3b affected the methylation of HOXA5. CpG islands in HOXA5 promoter region were analyzed via MethPrimer software (https://www.urogene.org) and MS-PCR showed that no methylation occurred at specific sites of HOXA5 in both the oe-NC and oeHOTAIR + sh-Dnmt3b groups, and methylation existed

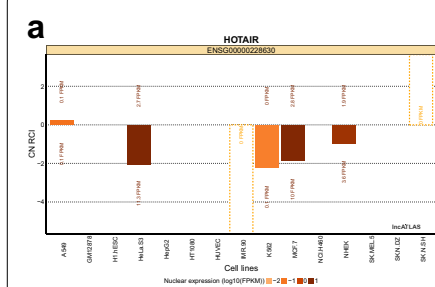

C
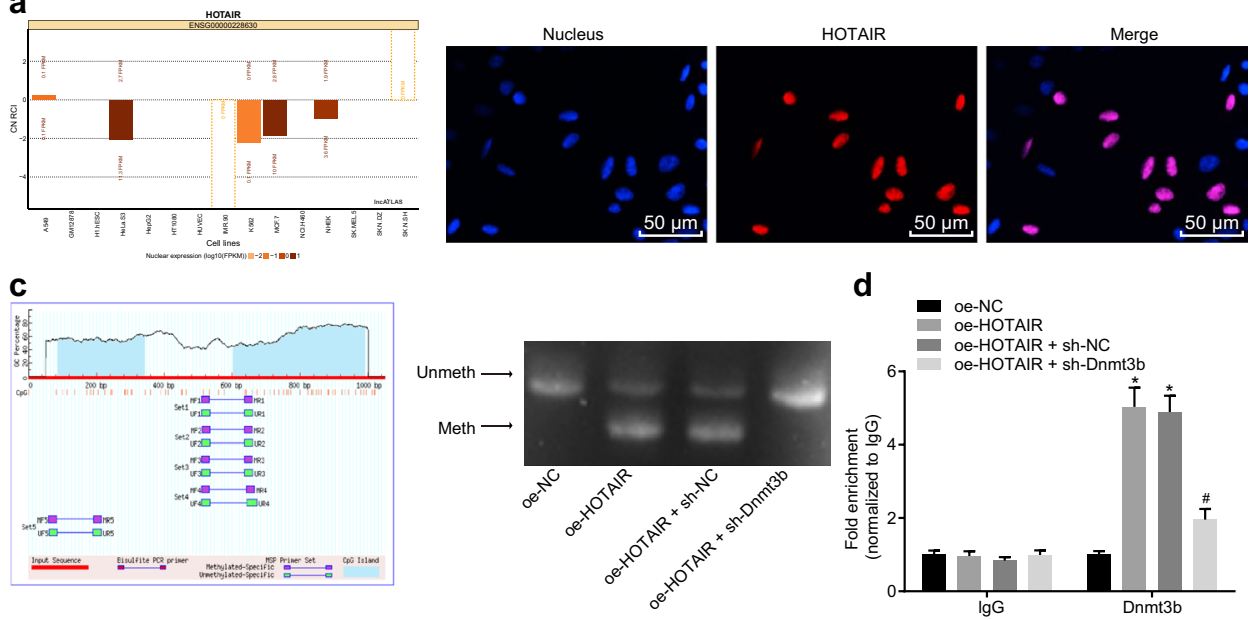

f
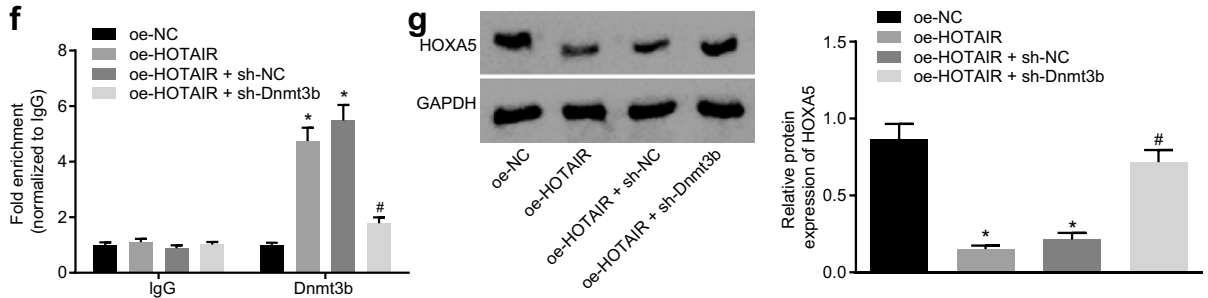

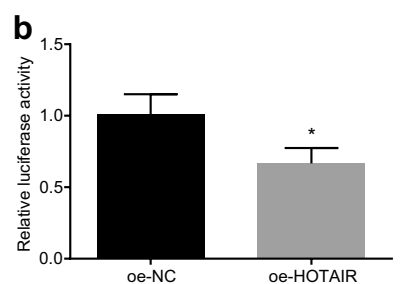

e

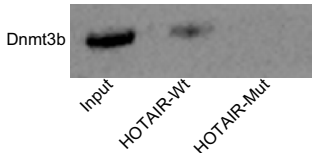

Fig. 4 HOTAIR and Dnmt3b downregulates HOXA5 through promoting its methylation. a The subcellular localization of HOTAIR predicted by IncATLAS website and verified by FISH $(\times 200)$. b Luciferase assay to confirm the binding of HOTAIR to HOXA5 promoter. c Distribution of CpG islands within the HOXA5 promoter region by MethPrimer and methylation level of HOXA5 promoter region detected by MSP. $\mathbf{d}$ The binding of Dnmt3b to HOTAIR after alteration of HOTAIR or Dnmt3b expression detected by RIP. e RNA pull down biotin-labeled HOTAIR and the expression of Dnmt3b in immunoprecipitation examined by western blot analysis. $\mathbf{f}$ The binding of Dnmt3b with HOXA5 promoter region after alteration of HOTAIR or Dnmt3b expression detected by CHIP-PCR. g Expression change of HOXA5 after alteration of HOTAIR or Dnmt3b expression examined by western blot analysis. ${ }^{*} p<0.05$ vs. the oe-NC group; ${ }^{*} p<0.05$ vs. the oe-HOTAIR + sh-NC group. RT- $q P C R$ reverse transcription quantitative polymerase chain reaction, NC negative control, FISH fluorescence in situ hybridization, RIP RNA immunoprecipitation, CHIP chromatin immunoprecipitation, HOTAIR Hox transcript antisense intergenic RNA, HOXA5 homeobox A5, Dnmt3b DNA methyltransferase 3b. The results were measurement data. Comparisons between two groups were conducted by means of independent sample t-test, and comparisons among multiple groups were assessed by one-way analysis of variance. The experiment was independently repeated three times 
at specific sites of HOXA5 in both the oe-HOTAIR and oe-HOTAIR + sh-NC groups (Fig. 4c), suggesting that HOTAIR could promote the methylation of HOXA5, which required Dnmt3b. It has also previously revealed that Dnmt3b-mediated DNA methylation was crucial in the progression of leukemia [21]. To further explore how Dnmt3b and HOTAIR function together to regulate HOXA5, RIP was used to detect whether Dnmt3b could bind with HOTAIR. It revealed that compared with the oe-NC group, the combination of HOTAIR and Dnmt3b was significantly increased in the oe-HOTAIR group $(p<0.05)$, and the combination of HOTAIR and Dnmt3b was significantly lower in the oe-HOTAIR + sh-Dnmt3b group than in the oe-HOTAIR + sh-NC group $(p<0.05$; Fig. 4d). RNA pull down assay was conducted to detect whether HOTAIR affected the degradation of Dnmt3b, and western blot analysis was used to examine the expression of Dnmt3b. It was found that the HOTAIR-Wt group showed decreased degradation of Dnmt3b expression while the HOTAIR-Mut group showed no Dnmt3b (Fig. 4e). Furthermore, CHIP was performed to detect whether Dnmt3b could directly bind to HOXA5 promoter region and whether HOTAIR affected the binding of Dnmt3b with HOXA5 promoter. Compared with the oe-NC group, Dnmt3b was remarkably enriched in HOXA5 promoter region in the oe-HOTAIR group. Compared with the oe-HOTAIR + sh-NC group, the oeHOTAIR + sh-Dnmt3b group showed reduced enrichment of Dnmt3b in HOXA5 promoter region $(p<0.05$; Fig. 4f). Western blot analysis showed that compared with the oe-NC group, the expression of HOXA5 in the oe-HOTAIR group was significantly decreased. In contrast to the oe-HOTAIR + sh-NC group, the expression of HOXA 5 in the oe-HOTAIR + sh-Dnmt3b group was notably increased $(p<0.05$; Fig. $4 \mathrm{~g})$. Taken together, HOTAIR, together with Dnmt3b, was able to promote the methylation of HOXA5 and then inhibited the expression of HOXA5.

\section{Silencing HOTAIR promotes apoptosis and represses proliferation of AML cells by elevating HOXA5 in vivo} In order to study the effects of HOTAIR and HOXA5 on HL-60 cells in vivo, we established xenograft tumor model in nude mice. Compared with the M-oe-NC group, the volume of tumor was significantly elevated in the oeHOTAIR group while was significantly decreased in the M-sh-HOTAIR group $(p<0.05)$. The volume of tumor in the M-oe-HOTAIR + oe-HOXA5 group was significantly lower than that in the M-oe-HOTAIR group $(p<0.05$; Fig. 5a). Then the levels of factors related to cell apoptosis were determined using western blot analysis. Figure $5 \mathrm{~b}$ showed that compared with the $\mathrm{M}$-oe-NC group, the expression of Bax, cleaved-caspase3, p27, cyclin G and HOXA5 was notably reduced while of $\mathrm{Bcl}-2$ and MCP-1 was obviously increased in the M-oe-HOTAIR group $(p<0.05)$. In contrast to the M-sh-NC group, the M-sh-HOTAIR group exhibited increased expression of Bax, cleaved-caspase3, p27, cyclin G and HOXA5 while decreased expression of Bcl-2 and MCP-1 $(p<0.05)$. The expression of Bax, cleaved-caspase3, p27, cyclin $\mathrm{G}$ and HOXA5 was notably higher while of Bcl-2 and MCP-1 was significantly lower in the M-oe-HOTAIR + oeHOXA5 group than those in the M-oe-HOTAIR group $(p<0.05$; Fig. $5 b)$. Lastly, EDU was conducted to detect the proliferation of HL- 60 cells, which revealed that compared with the M-oe-NC group, the cell proliferation in the M-oe-HOTAIR group was significantly elevated $(p<0.05)$. The cell proliferation was significantly reduced in the sh-HOTAIR group compared with the M-shNC group $(p<0.05)$. The cell proliferation in the M-oeHOTAIR + oe-HOXA5 group was significantly lower than that in the M-oe-HOTAIR group ( $p<0.05$; Fig. 5 c). The above results suggest that silencing HOTAIR could induce apoptosis and suppress proliferation of AML cells through up-regulating the expression of HOXA5.

\section{Determination of interference efficiency}

The interference efficiency of 3 independent shRAN against HOTAIR was examined. Compared with shHOTAIR-1 and sh-HOTAIR-2, the sh-HOTAIR-3 showed the highest silencing efficiency $(p<0.05$; Additional file 2: Fig. S1). Therefore, subsequent experiments were performed using the sh-HOTAIR-3 to silence HOTAIR.

\section{Discussion}

Despite the continuous improvement of AML treatment, prognosis of patient suffering from AML remains poor, partly due to the high relapse rate and the resistance to chemotherapy [22, 23]. DNA methylation pattern and gene expression profile of AML provides a new understanding in relation to the occurrence and development of AML, ultimately highlighting the potential of new research on novel therapeutic targets [24]. In recent years, HOTAIR, a type of IncRNA, has been demonstrated to participate in the progression of leukemia through regulating DNA methylation, as well as histones [25]. This study explored the potential roles of HOTAIR, HOXA5 methylation and Dnmt3b in AML cells, and got the conclusion that silencing HOTAIR could inhibit the methylation of HOXA5 via recruitment of Dnmt3b and effectively inhibit proliferation and induce apoptosis of AML cells.

Initially, the present study demonstrated that HOTAIR was highly expressed in AML cells. HOTAIR acts as a carcinogenic lncRNA, and elevated HOTAIR was found predict the poor overall survival in patients suffering from leukemia and lymphoma [26]. Moreover, another 


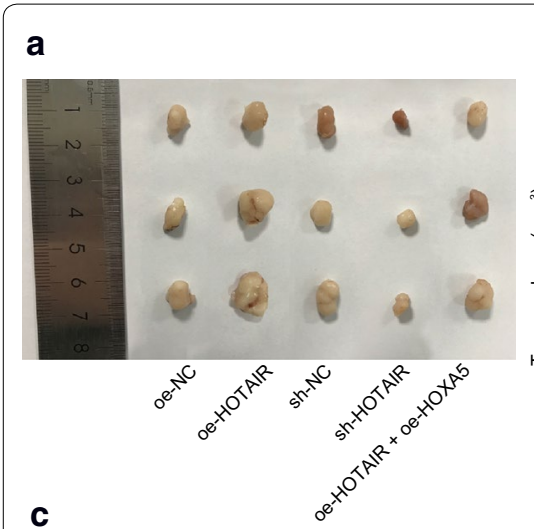

oe-NC
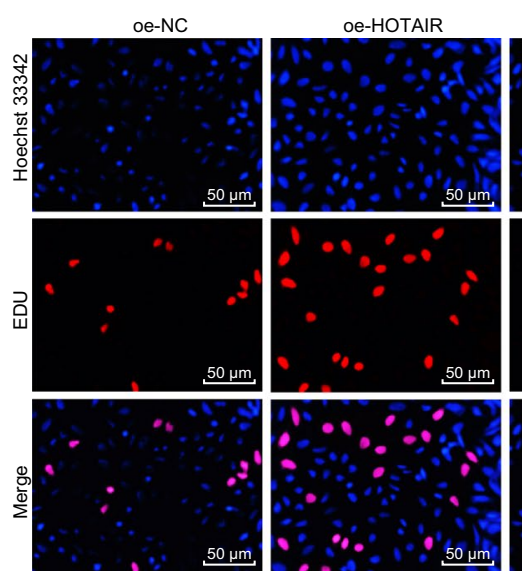
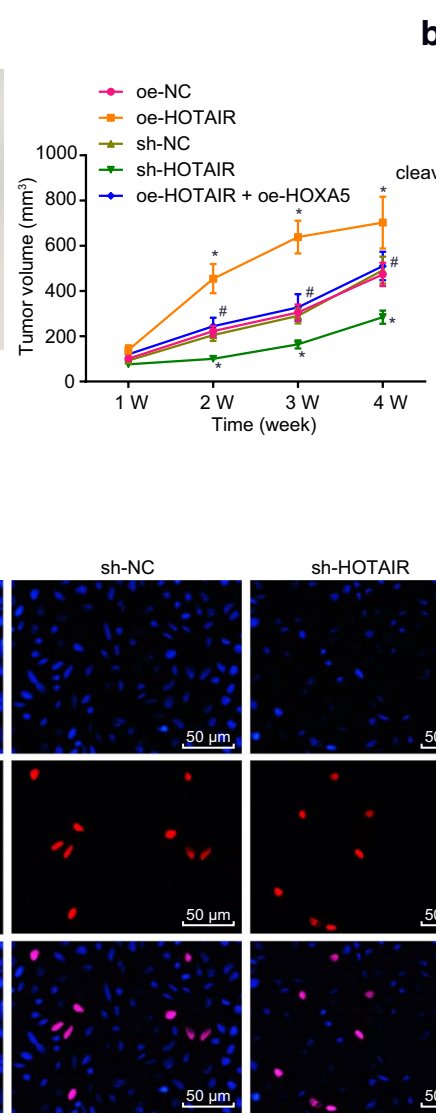

b
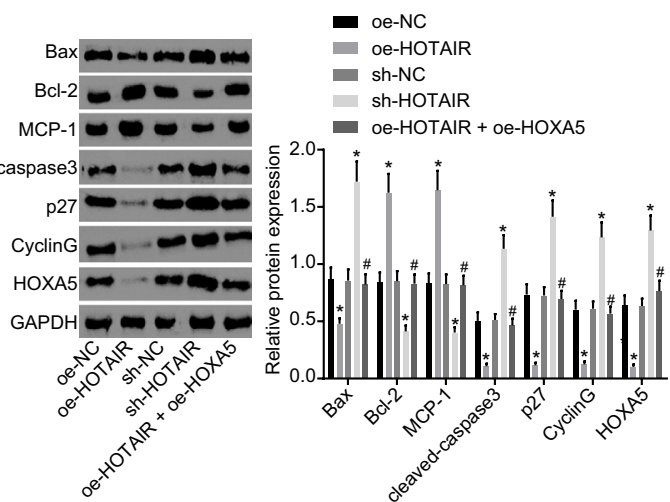

oe-HOTAIR

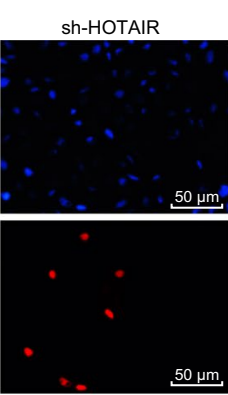
+ oe-HOXA5
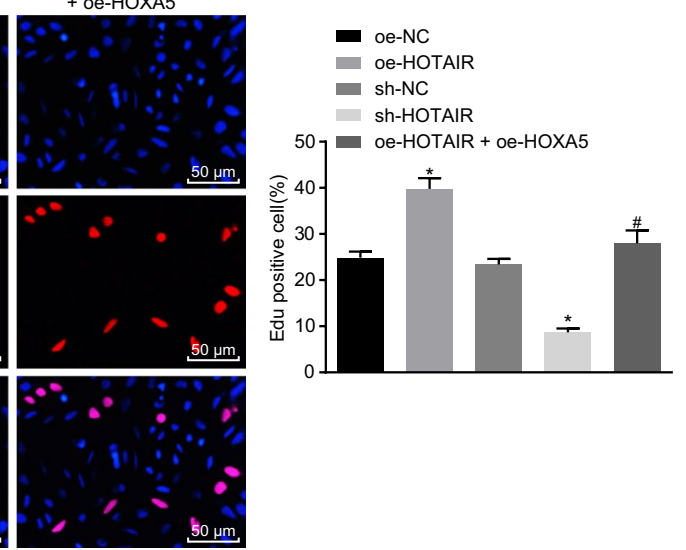

Fig. 5 Silenced HOTAIR enhances apoptosis and inhibits proliferation of HL-60 cells in vivo by upregulating HOXA5. a The volume of tumor from nude mice transplanted with $\mathrm{HL}-60$ cells overexpressing HOTAIR or HOXA5. b Expression of Bax, BCl-2, MCP-1, cleaved-caspase3, p27 and cyclin $\mathrm{G}$ in $\mathrm{HL}-60$ cell overexpressing HOTAIR or HOXA5 detected by western blot analysis. $\mathbf{c}$ EDU assay to evaluate the proliferation of HL-60 cells after overexpressing HOTAIR or HOXA5 ( $\times 200$ ). ${ }^{*} p<0.05$ vs. the M-oe-NC and M-sh-NC groups; ${ }^{*} p<0.05$ vs. the M-oe-HOTAIR and M-sh-HOTAIR groups. HOTAIR Hox transcript antisense intergenic RNA, HOXA5 homeobox A5, EDU 5-ethynyl-2'-deoxyuridine, BCl-2 B-cell lymphoma 2, Bax Bcl-2 associated $\mathrm{X}, \mathrm{MCP}-1$ monocyte chemoattractant protein 1, NCnegative control. The results were measurement data. Comparisons among multiple groups were assessed by one-way analysis of variance. The experiment was independently repeated three times

study has also indicated that HOTAIR is overexpressed in AML tissues [27]. Furthermore, in our study, downregulating HOTAIR was found to inhibit proliferation and promote apoptosis of AML cells, as supported by elevated Bax, cleaved-caspase3, p27 and cyclin G expressions in the AML cells transfected with sh-HOTAIR. The increased expression of Bax, cyclin D1, and cleaved-caspase 3 was related to induced cycle arrest and apoptosis of HL-60 acute leukemia cells [28]. Additionally, a prior study displayed that elevated expression of p27 could decline cell viability and induce cell apoptosis in CD44treated non-proliferating AML via forced expression of p27Kip1 [29]. Knockdown of HOTAIR triggered by small interfering RNA was previously suggested to suppress the proliferation of HL-60 and K562 cells in AML [12]. Besides, Wu et al. asserted that reduction of HOTAIR could induce apoptosis of K562-R cells in chronic myelogenous leukemia [30]. Furthermore, silencing HOTAIR by small hairpin RNA was found to suppress cell proliferation, induce apoptosis, and decline the colony formation ability in AML patients [31]. Collectively, our results concluded that HOTAIR might be an important target for the treatment of AML.

Additionally, our results found that HOTAIR was attributed to the methylation of the HOXA5 promoter, indicating that the function of HOTAIR in AML might be associated with HOXA5 promoter methylation. As a potential lncRNA biomarker in leukemia, HOTAIRM1 was identified to activate the temporal collinear HOXA gene, including HOXA5 [32]. HOXA5, a HOXA cluster gene, was reported to control the specification of myeloid and erythrocyte lineages, and its constitutive expression was identified to suppress erythropoiesis and induce the production of bone marrow cells [33]. HOTAIR was demonstrated to downregulate HOXA5 in lung cancer [13]. Overexpression of HOTAIR and methylation of HOXA5 


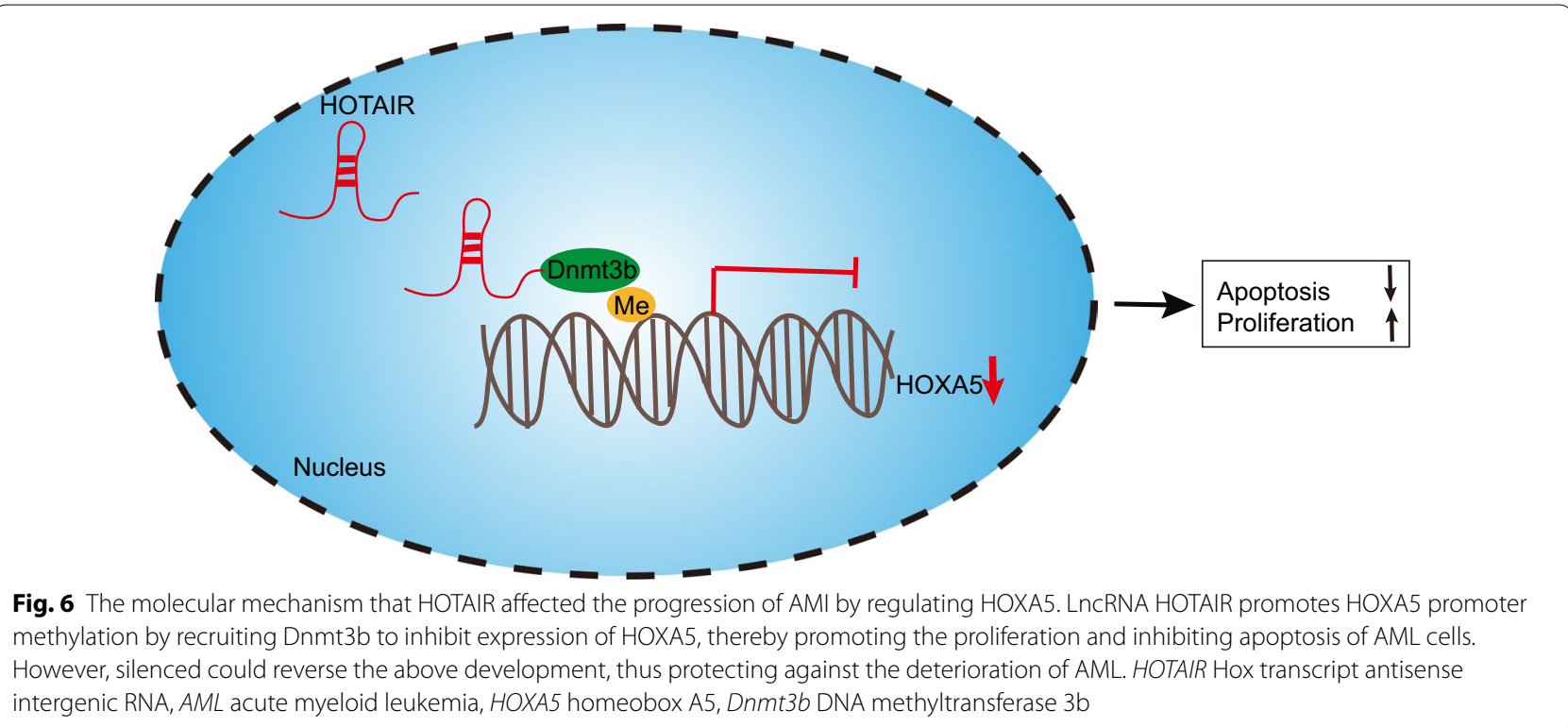

were also observed in the progression of breast cancer [19]. A previous study demonstrated that the hypermethylation of the HOXA5 promoter was related to the arrest of normal cell differentiation, thereby affecting the progression of AML [20]. In specific terms, HOTAIR was observed to promote the methylation of HOXA5 promoter by means of recruiting Dnmt3b. A previous study suggested that HOXA4 has an impact on the overall survival of AML by working with the DNA methylation protein (Dnmt1 or Dnmt3b) [34]. In addition, HOTAIR has been revealed to be correlated to upregulated Dnmt3a and Dnmt3b via the regulation of the DNA methylation in acute leukemia patients, contributing to the induced occurrence of acute leukemia [25]. Moreover, increased binding of Dnmt3b with pre-microRNA-375 (pre-miR-375) promoter could promote the DNA hypermethylation of pre-miR-375 and decline expression of miR-375, thereby leading to leukemogenesis in AML [35]. Interestingly, our study presented that after overexpression of HOXA5, cell proliferation was decreased and cell apoptosis was increased. Consistent with our results, Xudong Peng et al. observed that HOXA5 inhibited GC cell proliferation in vivo [36]. In addition, another study also found that HOXA5 abrogated cell apoptosis induced by physcion, which is in line with our study [37].

\section{Conclusion}

In summary, the key findings of the study revealed that HOTAIR was overexpressed in AML and that silencing of HOTAIR repressed methylation of HOXA5 promoter by decreasing Dnmt3b, thereby inhibiting proliferation and inducing apoptosis of AML cells (Fig. 6). Based on the evidence of this study, therapeutic strategies should be directed towards the down-regulation of HOTAIR, which may potentially be a clinically viable target in the treatment of AML.

\section{Additional files}

Additional file 1: Table S1. The clinical characteristics of patients.

Additional file 2: Figure S1. The interference efficiency of different shRNA against HOTAIR detected by RT-qPCR. ${ }^{*} p<0.05$ vs. the sh-NC group; ${ }^{p}<0.05$ vs. the sh-HOTAIR-1 and sh-HOTAIR-2 sequences; RT-qPCR, reverse transcription quantitative polymerase chain reaction; HOTAIR, Hox transcript antisense intergenic RNA; NC, negative control. The results were measurement data. Comparisons between two groups were conducted by means of independent sample $t$-test. The experiment was independently repeated three times.

\section{Abbreviations}

AML: acute myelogenous leukemia; FLT3: fetal liver tyrosine kinase 3; ITD: internal tandem duplication; IncRNAs: long non-coding RNAs; HOTAIR: homeobox antisense intergenic RNA; HOXA5: homeobox A5; Dnmt3b: DNA methyltransferase 3b; WHO: World Health Organization; POX: peroxidase; ANAE: alpha-naphthyl-acetate-esterase; CE: specific esterase; PAS: periodic acid-Schiff; RT-qPCR: reverse transcription quantitative polymerase chain reaction; NC: negative control; GAPDH: glyceraldehyde-3-phosphate dehydrogenase; RIPA: radioimmunoprecipitation assay; BCA: bicinchoninic acid; PVDF: polyvinylidene fluoride; BSA: bovine serum albumin; ECL: enhanced chemiluminescence; FISH: fluorescence in situ hybridization; PBS: phosphate-buffered saline; MS: methylation specific; CT: cytosine to thymine; pRL: promoter-renilla luciferase; TK: thymidine kinase; RIP: RNA immunoprecipitation; WT: wild-type; BSA: bovine serum albumin; CHIP: chromatin immunoprecipitation; FITC: fluorescein isothiocyanate; PI: propidium iodide; EDU: 5-ethynyl-2'-deoxyuridine; SPF: specific pathogen-free.

\section{Authors' contributions}

HY designed the study. SR collated the data, designed and developed the database. YYY carried out data analyses and produced the initial draft of the 
manuscript. WSL contributed to drafting the manuscript. All authors read and approved the final manuscript.

\section{Author details}

${ }^{1}$ Department of Hematology, The First Affiliated Hospital of Xiamen University, No. 55, Zhenhai Road, Xiamen 361003, Fujian, People's Republic of China. 2 Department of Clinical Medicines, Fujian Medical University, No. 1, Xuefu North Road, Fuzhou 350108, Fujian, People's Republic of China. ${ }^{3}$ Department of General Surgery, The First Affiliated Hospital of Xiamen University, Xiamen 361003, People's Republic of China.

\section{Acknowledgements}

We acknowledge and appreciate our colleagues for their valuable efforts and comments on this paper.

\section{Competing interests}

The authors declare that they have no competing interests.

\section{Availability of data and materials}

The datasets generated and/or analyzed during the current study are available from the corresponding author on reasonable request.

\section{Consent for publication}

Not applicable.

\section{Ethics approval and consent to participate}

The present study was conducted in strict accordance to the protocols approved by the Institutional Review Board of The First Affiliated Hospital of Xiamen University. Written informed consent documentations were signed by all participating patients. All animal experiments were performed in full compliance with the national principles for animal usage in research with the approval of the Animal Care and Use Committee. All animal experiments in this study were in conformity to the guidelines of management and use of local laboratory animals and approved by the Guide for the Care and Use of Laboratory Animal issued by the National Institutes of Health.

\section{Funding}

This study was supported by Natural Science Foundation of Fujian Province in 2018 (2018J01387) and Xiamen Medical and Health project Foundation (Guiding project) in 2018 (3502Z20189005).

\section{Publisher's Note}

Springer Nature remains neutral with regard to jurisdictional claims in published maps and institutional affiliations.

Received: 3 November 2018 Accepted: 28 March 2019

Published online: 29 April 2019

\section{References}

1. Somintara S, Leardkamolkarn V, Suttiarporn P, et al. Anti-tumor and immune enhancing activities of rice bran gramisterol on acute myelogenous leukemia. PLOS ONE. 2016;11:e0146869.

2. Siveen KS, Uddin S, Mohammad RM. Targeting acute myeloid leukemia stem cell signaling by natural products. Mol Cancer. 2017;16:13.

3. Bi L, Sun $L$, Jin $Z$, et al. MicroRNA-10a/b are regulators of myeloid differentiation and acute myeloid leukemia. Oncol Lett. 2018;15:5611-9.

4. Yanada M, Matsuo K, Emi N, et al. Efficacy of allogeneic hematopoietic stem cell transplantation depends on cytogenetic risk for acute myeloid leukemia in first disease remission: a metaanalysis. Cancer. 2005;103:1652-8.

5. Wang X, Chen X, Yang Z, et al. Correlation of TET2 SNP rs2454206 with improved survival in children with acute myeloid leukemia featuring intermediate-risk cytogenetics. Genes Chromosomes Cancer. 2018;57:379-86

6. Mambet C, Chivu-Economescu M, Matei L, et al. Murine models based on acute myeloid leukemia-initiating stem cells xenografting. World J Stem Cells. 2018;10:57-65.
7. Freedman JE, Miano JM, National Heart L, et al. Challenges and opportunities in linking long noncoding RNAs to cardiovascular, lung, and blood diseases. Arterioscler Thromb Vasc Biol. 2017:37:21-5.

8. Hirano T, Yoshikawa R, Harada H, et al. Long noncoding RNA, CCDC26, controls myeloid leukemia cell growth through regulation of KIT expression. Mol Cancer. 2015;14:90

9. Jia ZW, Li Y, Cui GR, et al. Expression and clinical significance of LncRNA KCNQ1OT1 in patients with acute myeloid leukemia. Zhongguo Shi Yan Xue Ye Xue Za Zhi. 2018;26:653-7.

10. Diaz-Beya M, Brunet $S$, Nomdedeu J, et al. The lincRNA HOTAIRM1, located in the HOXA genomic region, is expressed in acute myeloid leukemia, impacts prognosis in patients in the intermediate-risk cytogenetic category, and is associated with a distinctive microRNA signature. Oncotarget. 2015;6:31613-27.

11. Wu S, Zheng C, Chen S, et al. Overexpression of long non-coding RNA HOTAIR predicts a poor prognosis in patients with acute myeloid leukemia. Oncol Lett. 2015;10:2410-4.

12. Hao S, Shao Z. HOTAIR is upregulated in acute myeloid leukemia and that indicates a poor prognosis. Int J Clin Exp Pathol. 2015;8:7223-8.

13. Abba MC, Gong T, Lu Y, et al. A molecular portrait of high-grade ductal carcinoma in situ. Cancer Res. 2015;75:3980-90.

14. Liu XH, Liu ZL, Sun M, et al. The long non-coding RNA HOTAIR indicates a poor prognosis and promotes metastasis in non-small cell lung cancer. BMC Cancer. 2013;13:464.

15. Avraham A, Sandbank J, Yarom N, et al. A similar cell-specific pattern of HOXA methylation in normal and in cancer tissues. Epigenetics. 2010;5:41-6.

16. Li N, Jia X, Wang J, et al. Knockdown of homeobox A5 by small hairpin RNA inhibits proliferation and enhances cytarabine chemosensitivity of acute myeloid leukemia cells. Mol Med Rep. 2015;12:6861-6.

17. Niederwieser C, Kohlschmidt J, Volinia S, et al. Prognostic and biologic significance of DNMT3B expression in older patients with cytogenetically normal primary acute myeloid leukemia. Leukemia. 2015;29:567-75

18. Raju Bagadi SA, Kaur J, Ralhan R. Establishment and characterisation of two novel breast cancer cell lines. Cell Biol Int. 2008;32:55-65.

19. Mohammadali F, Abroun S, Atashi A. Mild hypoxia and human bone marrow mesenchymal stem cells synergistically enhance expansion and homing capacity of human cord blood CD34+ stem cells. Iran J Basic Med Sci. 2018;21:709-16.

20. Strathdee G, Sim A, Soutar R, et al. HOXA5 is targeted by cell-type-specific CpG island methylation in normal cells and during the development of acute myeloid leukaemia. Carcinogenesis. 2007;28:299-309.

21. Schulze I, Rohde C, Scheller-Wendorff M, et al. Increased DNA methylation of Dnmt3b targets impairs leukemogenesis. Blood. 2016;127:1575-86

22. Cruz NM, Mencia-Trinchant N, Hassane DC, et al. Minimal residual disease in acute myelogenous leukemia. Int J Lab Hematol. 2017;39(Suppl 1):53-60.

23. Ding $\mathrm{YH}$, Gao X, Long J, et al. Dispirocyclopropyldehydrocostus lactone selectively inhibits acute myelogenous leukemia cells. Bioorg Med Chem Lett. 2016;26:1165-8.

24. Schoofs T, Berdel WE, Muller-Tidow C. Origins of aberrant DNA methylation in acute myeloid leukemia. Leukemia. 2014;28:1-14.

25. Zhang YY, Huang SH, Zhou HR, et al. Role of HOTAIR in the diagnosis and prognosis of acute leukemia. Oncol Rep. 2016;36:3113-22.

26. Lin Y, Fang Z, Lin Z, et al. The prognostic impact of long noncoding RNA HOTAIR in leukemia and lymphoma: a meta-analysis. Hematology. 2018;23:600-7

27. Sayad A, Hajifathali A, Hamidieh AA, et al. HOTAIR Long Noncoding RNA is not a Biomarker for Acute Myeloid Leukemia (AML) in Iranian Patients. Asian Pac J Cancer Prev. 2017;18:1581-4.

28. Yang G, Yang L, Zhuang Y, et al. Ganoderma lucidum polysaccharide exerts anti-tumor activity via MAPK pathways in HL-60 acute leukemia cells. J Recept Signal Transduct Res. 2016;36:6-13.

29. Gadhoum Z, Leibovitch MP, Qi J, et al. CD44: a new means to inhibit acute myeloid leukemia cell proliferation via p27Kip1. Blood. 2004:103:1059-68.

30. Wang H, Li Q, Tang S, et al. The role of long noncoding RNA HOTAIR in the acquired multidrug resistance to imatinib in chronic myeloid leukemia cells. Hematology. 2017;22:208-16. 
31. Xing CY, Hu XQ, Xie FY, et al. Long non-coding RNA HOTAIR modulates C-KIT expression through sponging miR-193a in acute myeloid leukemia. FEBS Lett. 2015;589:1981-7.

32. Wang $X Q$, Dostie J. Reciprocal regulation of chromatin state and architecture by HOTAIRM1 contributes to temporal collinear HOXA gene activation. Nucleic Acids Res. 2017;45:1091-104.

33. Musialik E, Bujko M, Kober $P$, et al. Promoter DNA methylation and expression levels of HOXA4, HOXA5 and MEIS1 in acute myeloid leukemia. Mol Med Rep. 2015:11:3948-54.

34. Grubach L, Juhl-Christensen C, Rethmeier A, et al. Gene expression profiling of Polycomb, Hox and Meis genes in patients with acute myeloid leukaemia. Eur J Haematol. 2008:81:112-22.
35. Bi L, Zhou B, Li H, et al. A novel miR-375-HOXB3-CDCA3/DNMT3B regulatory circuitry contributes to leukemogenesis in acute myeloid leukemia. BMC Cancer. 2018;18:182

36. Peng $X$, Zha $L$, Chen $A$, et al. HOXA5 is a tumor suppressor gene that is decreased in gastric cancer. Oncol Rep. 2018;40:1317-29.

37. Gao F, Liu W, Guo Q, et al. Physcion blocks cell cycle and induces apoptosis in human B cell precursor acute lymphoblastic leukemia cells by downregulating HOXA5. Biomed Pharmacother. 2017;94:850-7.
Ready to submit your research? Choose BMC and benefit from:

- fast, convenient online submission

- thorough peer review by experienced researchers in your field

- rapid publication on acceptance

- support for research data, including large and complex data types

- gold Open Access which fosters wider collaboration and increased citations

- maximum visibility for your research: over $100 \mathrm{M}$ website views per year

At BMC, research is always in progress.

Learn more biomedcentral.com/submissions 